\title{
CARACTERIZAÇÃO DE ESPUMAS DE FLOTAÇÃO VIA ARDUINO
}

Felipe de Orquiza Milhomem - felipe.milhomem6@gmail.com

Programa de Pós-Graduação em Engenharia Mineral, Departamento de Engenharia de Minas, Universidade Federal de Ouro Preto

Campus Morro do Cruzeiro

CEP 35400-000 - Ouro Preto - Minas Gerais

José Aurélio Medeiros da Luz-jaurelio@ufop.edu.br

Programa de Pós-Graduação em Engenharia Mineral, Departamento de Engenharia de Minas, Universidade Federal de Ouro Preto

Campus Morro do Cruzeiro

CEP 35400-000 - Ouro Preto - Minas Gerais

Resumo: A flotação é uma operação de concentração muito utilizada no beneficiamento mineral. Ela faz a separação entre minerais a partir da atuação de reagentes tensoativos que modificam as superfícies dos sólidos, podendo torna-los hidrofóbicos seletivamente. Ela é realizada em meio líquido e com inserção de ar, de forma que o sólido hidrofobizado adira às bolhas de ar. Sendo um parâmetro de grande importância no processo, seu controle deve ser bem realizado, sendo a determinação da fração volumétrica de ar, ou holdup, uma das variáveis mais importantes. Sua determinação usualmente é feita pela medição da diferença de pressão entre dois pontos do equipamento, embora também possa ser feita através de medidas de condutividade. Assim, desenvolveu-se um sistema de baixo custo para medir a condutividade elétrica em um sistema de flotação, através do uso da plataforma Arduino. Os resultados mostraram que o sistema se mostrou eficaz para medições condutividade, sendo uma proposta tanto para implementar aprendizado multidisciplinar quanto para aquisição de dados em laboratório com baixo custo.

Palavras-chave: Arduino. flotação. fração de ar, condutividade elétrica, baixo custo. 


\section{INTRODUÇÃO}

A flotação é uma das operações mais utilizadas no beneficiamento mineral, dada sua versatilidade e possibilidade de processar partículas muito finas (tamanhos menores que $150 \mu \mathrm{m}$ ), em faixas nas quais outras operações encontram algumas dificuldades. Basicamente, a flotação é um processo físico-químico no qual a superfície dos sólidos é alterada artificialmente pelo uso de reagentes tensoativos, tornando-os hidrofóbicos. A inserção de ar no sistema leva à adesão dos sólidos hidrofobizados nas bolhas de ar, que os transportam para a parte superior do equipamento, onde serão coletados. É possível obter então dois produtos, o flotado e o não flotado (aquele sólido que não foi hidrofobizado seletivamente).

Além dos reagentes que garantem a hidrofobicidade mineral, existem aqueles denominados espumantes, que como o nome sugere, auxiliam na formação de uma espuma rica e estável que facilite o transporte dos sólidos. Com isso, a aeração é um parâmetro muito importante no processo. Embora seja possível saber a vazão de ar que alimenta o equipamento, a quantificação da fração volumétrica de ar, também chamado holdup, presente na camada de espuma, é mais difícil.

Dentre os métodos de mensuração da fração de ar, existe o condutivimétrico, que como o nome sugere, utiliza da medida da condutividade elétrica para a quantificação da vazão de ar.

Pensando na possibilidade da interdisciplinaridade do processamento mineral com conceitos intrínsecos à eletrônica e programação, aliado à opção de uso de plataformas de código aberto e baixo custo, propôs-se aqui o uso da placa microcontroladora Arduino, modelo UNO, para construção de um condutivímetro que permita medir a fração volumétrica de gás em equipamentos de flotação.

\section{REVISÃO BIBLIOGRÁFICA}

Existem diversas técnicas para medição das condições vigentes em reatores, sejam eles compostos por sistemas bifásicos (líquido e gás) ou trifásico (sólido, líquido e gás). Boyer, Duquenne e Wild (2002) apresentam algumas dessas técnicas, com os sistemas nas quais podem ser aplicadas e resultados que podem ser obtidos, como fração do líquido ou gás, turbulência, queda de pressão, vazão, distribuição de fases, distribuição do tamanho de bolhas, entre outros. $\mathrm{Na}$ "Tabela 1" encontram-se alguns desses métodos.

No caso dos sistemas de flotação, a fração do gás é uma importante variável de processo por permitir estimar tamanho de bolhas e controlar a interface da espuma no equipamento. Ele depende, dentre outras coisas, da vazão de ar, da densidade da polpa e do carregamento dos sólidos nas bolhas (SASTRI, 1998; AQUINO; OLIVEIRA; FERNANDES, 2010). Ele é normalmente determinado através da diferença de pressão (BAHRI; SHAFAEI; KARAMOOZIAN, 2013; JENA; ROY; MEIKAP, 2008; PAN et al., 2018; TAVERA; ESCUDERO; FINCH, 2001) com uso da "Equação (1)", a seguir:

$$
\varepsilon_{g}=\frac{1-\Delta P}{\rho_{p o} \times g \times \Delta H}
$$

Onde:

$\varepsilon_{\mathrm{g}}$ - fração volumétrica do gás [-];

$\rho_{\mathrm{po}}-$ massa específica da polpa $\left[\mathrm{kg} / \mathrm{m}^{3}\right]$ 
$\Delta \mathrm{P}$ - diferença de pressão entre dois pontos no equipamento [Pa];

$\mathrm{g}$ - aceleração da gravidade $\left[\mathrm{m} / \mathrm{s}^{2}\right]$;

$\Delta \mathrm{H}$ - distância entre os dois pontos de medição [m].

Tabela 1 - Algumas técnicas não invasivas para análise de reatores.

\begin{tabular}{|l|l|l|}
\hline \multicolumn{1}{|c|}{ Técnica } & \multicolumn{1}{|c|}{ Aplicação } & \multicolumn{1}{c|}{ Resultados obtidos } \\
\hline Queda de pressão & GL e GLS & $\begin{array}{l}\text { Queda de pressão, regime } \\
\text { hidrodinâmico, fração do } \\
\text { líquido }\end{array}$ \\
\hline Condutimetria & GL e GLS & Fração volumétrica \\
\hline Ultrassom & GL e GLS com restrições & Fração volumétrica \\
\hline Análise de imagens & Principalmente GL & $\begin{array}{l}\text { Tamanho e forma de bolhas, } \\
\text { regime hidrodinâmico }\end{array}$ \\
\hline Traçadores radioativos & GL e GLS & $\begin{array}{l}\text { Velocidade e trajetória do } \\
\text { fluxo }\end{array}$ \\
\hline Tomografia ultrassônica & GL e GLS & $\begin{array}{l}\text { Distribuição da fração } \\
\text { volumétrica das fases }\end{array}$ \\
\hline Radiografia & Principalmente GL & $\begin{array}{l}\text { Tamanho e forma de bolhas, } \\
\text { regime hidrodinâmico }\end{array}$ \\
\hline
\end{tabular}

Fonte: modificado de Boyer, Duquenne e Wild (2002).

GL - gás-líquido; GLS - gás-líquido-sólido.

Entretanto, também pode-se utilizar de medidas de condutividade para determinação da fração volumétrica de gás. Para isso, utiliza-se a equação de Maxwell (TAVERA; GOMEZ; FINCH, 1998; URIBE-SALAS; GOMEZ; FINCH, 1994; XU; FINCH; HULS, 1992), dada pela "Equação (2)":

$$
\varepsilon_{g}=\frac{1-\left(k_{d} / k_{p o}\right)}{1+\left(0,5 \times k_{d} / k_{p o}\right)}
$$

Onde:

$\kappa_{d}-$ condutividade da dispersão (polpa aerada) $[\mathrm{mS} / \mathrm{m}]$;

$\kappa_{\mathrm{po}}-$ condutividade da polpa (sem aeração) $[\mathrm{mS} / \mathrm{m}]$.

Uribe-Salas, Gomez e Finch (1994) fizeram estudos comparando a predição da fração do ar por medidas condutivimétricas com aquelas por diferença de pressão, e encontraram boa correlação entre ambos os modelos. 


\section{MATERIAIS E MÉTODOS}

\subsection{Construção e programação da sonda}

O princípio utilizado para a construção da sonda foi o de divisor de tensão, de acordo com a Segunda Lei de Kirchhoff (TORRES, 2002). Na "Figura 1" é apresentado seu esquema, com uma das resistências (R1) sendo do meio (no caso em questão, a espuma trifásica mineralizada), e a outra (R2) um resistor de valor conhecido. A tensão de entrada é aquela fornecida ao sistema, dada por $\mathrm{V}_{\text {in }}$ e a tensão de saída, ou de leitura, dada por $\mathrm{V}_{\text {out. }} \mathrm{O}$ sistema é ainda ligado ao terra, sendo mostrado pelo símbolo de seta no inferior da figura.

\section{Figura 1 - Divisor de tensão.}

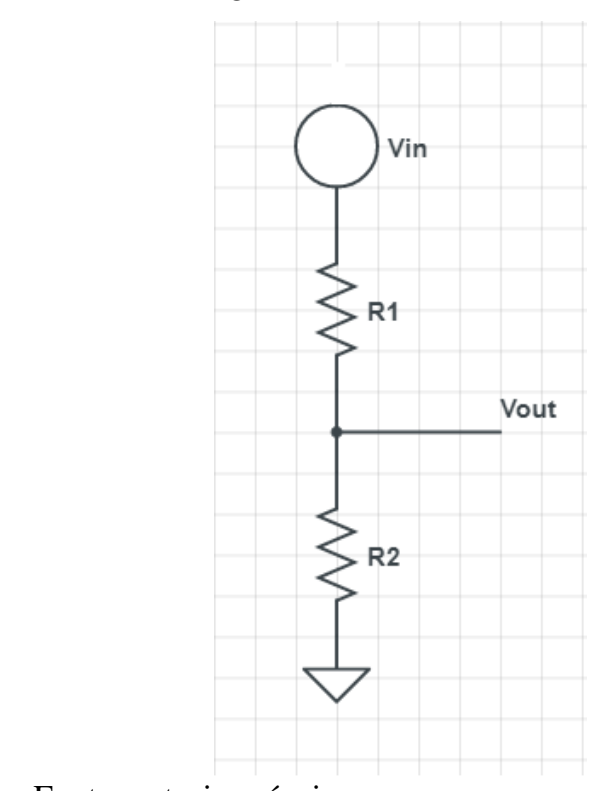

Fonte: autoria própria

A sonda é alimentada pelo pino de $5 \mathrm{~V}$ do Arduino ( $\mathrm{V}_{\text {in }}$ ) e a tensão de saída (ou retorno) é lida pelo seu pino analógico. Com esse valor, é possível calcular a resistência do meio pela "Equação (3)":

$$
R_{\text {meio }}=\frac{\left(V_{\text {in }} \times R_{c}\right)}{V_{\text {out }}}-R_{c}
$$

Sendo:

$\mathrm{R}_{\text {meio }}$ - valor de resistência procurado (resistência do meio) [ $\Omega$ ];

$\mathrm{R}_{\mathrm{c}}$ - resistência de controle, ou seja, um resistor de valor conhecido [ $\left.\Omega\right]$;

$\mathrm{V}_{\text {in }}$ - tensão oferecida pelo Arduino [5 V];

$\mathrm{V}_{\text {out }}$ - tensão de saída, ou seja, a tensão lida pelo Arduino [ $\left.\Omega\right]$.

A placa Arduino possui portas digitais, que diferenciam dois níveis (HIGH e LOW), e analógicas, que diferem 1.024 níveis de voltagem diferentes ( 0 a 1023). Ao se usar a porta analógica, o Arduino UNO realiza cada leitura como 5 volts/1.024, ou 0,0049 V. Ou seja, será realizada uma leitura entre 0 e 1.023 que representa entre 0 e 5 volts (BANZI; SHILOH, 2015). 
Uma correção deve ser feita para a conversão do sinal (TEIXEIRA; SANTOS, 2013). Com isso, a leitura da tensão de saída é dada pela "Equação (4)":

$$
V_{\text {out }}=\frac{V_{\text {in }}}{1023} \times V_{\text {lido }}
$$

Sendo $\mathrm{V}_{\text {lido }}$ a tensão lida pela entrada analógica do Arduino. De posse da resistência do meio, é possível convertê-la para encontrar a condutividade elétrica.

Embora existam opções de compra de células de condutividade que podem ser conectadas ao Arduino, a exemplo da disponível em DFRobot (2020), aqui optou-se pela elaboração de sonda própria. Utilizou-se um plugue de tomada tipo A, com pinos achatados. Para o cálculo da condutividade, é preciso ter a constante de célula, que depende das características geométricas do eletrodo. A área dos eletrodos foi calculada com uso de paquímetro e sua constante de célula, neste caso, apresentou valor de 1,791359. A montagem do sistema placa Arduino/sonda pode ser vista na "Figura 2".

Figura 2 - Placa Arduino montada com a sonda condutivimétrica.

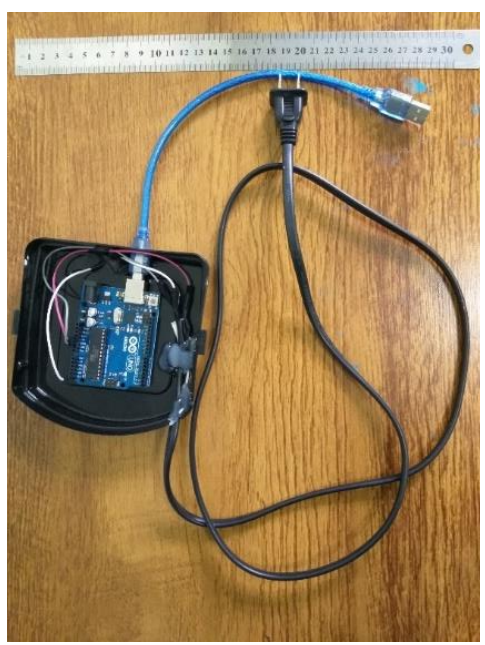

Fonte: autoria própria.

Já na "Figura 3" encontra-se o algoritmo básico para a leitura dos valores obtidos para leitura da condutividade elétrica pelo Arduino.

Figura 3 - Algoritmo do Arduino para leitura dos valores de resistência e cálculo da condutividade. 


\subsection{Ensaios de flotação}

Embora o objetivo primordial da flotação seja a separação dos minerais presentes na polpa entre o material de valor econômico (dito concentrado) e o sem valor econômico (denominado rejeito), neste estudo optou-se por se realizar a flotação apenas de um mineral, a hematita. Isto pois o objetivo é somente a avaliação da condutividade da espuma, e não de otimização de sistemas de flotação.

Foi utilizada uma cuba (recipiente) de flotação de $0,0008 \mathrm{~m}^{3}$ de volume útil $(800 \mathrm{ml})$, com porcentagem de sólidos de $40 \%$. O pH da polpa foi fixado em 8,0, com seu controle feito por meio de $\mathrm{HCl}$ e $\mathrm{NaOH}$. O reagente coletor utilizado foi o 9-octadecenoato de sódio, comumente denominado oleato de sódio, na dosagem de $500 \mathrm{~g} / \mathrm{t}$, obtido pela saponificação do ácido oleico com excesso de $\mathrm{NaOH}$ alcoólico a $0,25 \mathrm{M}$. Este excesso se deu até a solução atingir pH maior que 12. Maiores informações sobre processo de saponificação podem ser vistos em Oliveira, Luz e Ferreira (2006). Foi utilizado óleo de pinho como reagente tensoativo (espumante) para garantir maior estabilidade à espuma. $\mathrm{O}$ procedimento foi realizado com as seguintes etapas:

1. A massa desejada de sólidos e água era colocada na cuba de flotação;

2. Após breve agitação para homogeneização, seu pH era medido;

3. O coletor era adicionado na polpa, o pH medido novamente e modificado para o valor desejado;

4. A polpa era posta em agitação por 7 minutos, denominado tempo de condicionamento, no qual os reagentes agem sobre a superfície mineral;

5. No último minuto do tempo de condicionamento, 3 gotas de espumante eram adicionadas na polpa;

6. A vazão de ar era liberada e a espuma mineralizada transbordante era coletada, em intervalos de 30 segundos, durante 120 segundos;

7. Finalizado este tempo, a rotação do rotor e a vazão de ar eram desligados;

8. Os recipientes de coleta eram pesados ainda com a polpa, postos para secar a $105{ }^{\circ} \mathrm{C}$ por uma hora, e pesados novamente.

Embora uma prática laboratorial comum em ensaios de flotação seja a reposição de água ao longo do experimento, optou-se aqui por evitar tal prática. Com isso, o controle da água ficou mais fácil, apenas pelas diferenças entre as massas úmidas e secas.

Ressalte-se ainda que a sonda do Arduino ficava conectada durante todo o tempo de flotação, a uma altura fixa, no sentido do transbordo da espuma, e de forma que ela ficasse em contato com a fase sólido/líquido/gás e não somente na fase sólido/líquido. Na "Figura 4" encontra-se a célula de flotação usada, bem como a inserção da sonda na mesma.

Foi medida a condutividade da polpa, sem aeração, e também durante a flotação, com a polpa aerada. A fração de ar foi calculada com base na "Equação (2)". Também foi feita comparação da evolução da condutividade elétrica com a massa total flotada (sólido + líquido). 
Figura 4 - À esquerda, a célula de flotação; à direita, detalhes da coleta da espuma mineralizada e o posicionamento da sonda condutivimétrica.
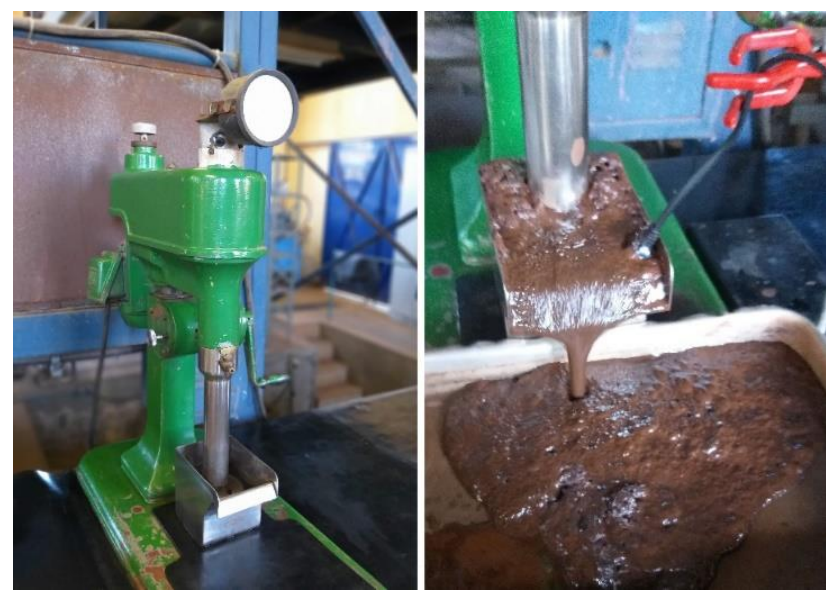

Fonte: autoria própria.

\section{RESULTADOS E DISCUSSÕES}

$\mathrm{Na}$ "Figura 5", encontram-se a comparação entre a recuperação mássica de sólidos + líquido com a condutividade elétrica, através do tempo de operação. Como não houve adição de líquido no decorrer do ensaio, sua massa foi calculada com base na diferença entre a massa de sólidos seca e a massa do prato pesado após a coleta. Pela figura em questão, percebe-se o efeito esperado da redução da condutividade no decorrer do ensaio, devido à redução de material, ou seja, redução de material disponível no recipiente de flotação. Padrão semelhante a esse foi demonstrado no trabalho de Zhu, Tan e Gleisner (2005), no qual a condutividade também decaia com o decorrer da operação de flotação. Resultado

Figura 5 - Comparação da condutividade elétrica com a recuperação mássica para dois ensaios de flotação.

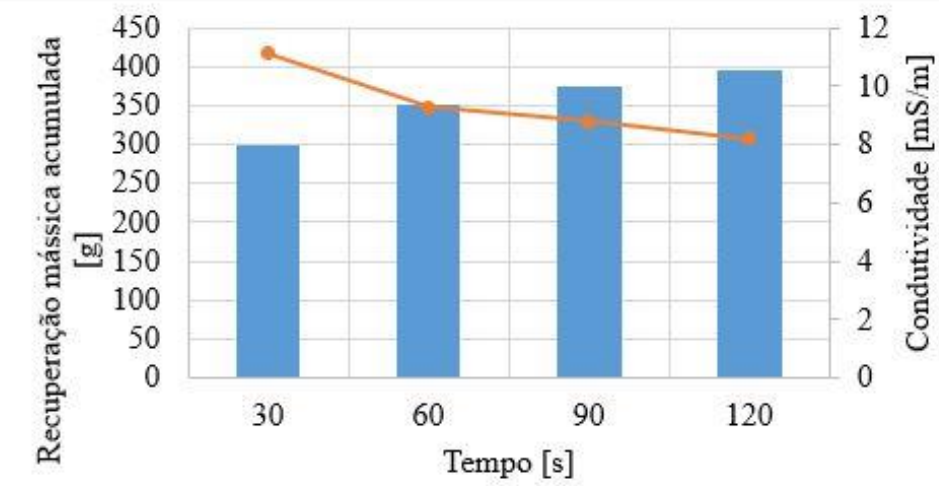

Recuperação mássica [sólidos + água] $\rightarrow$ Condutividade

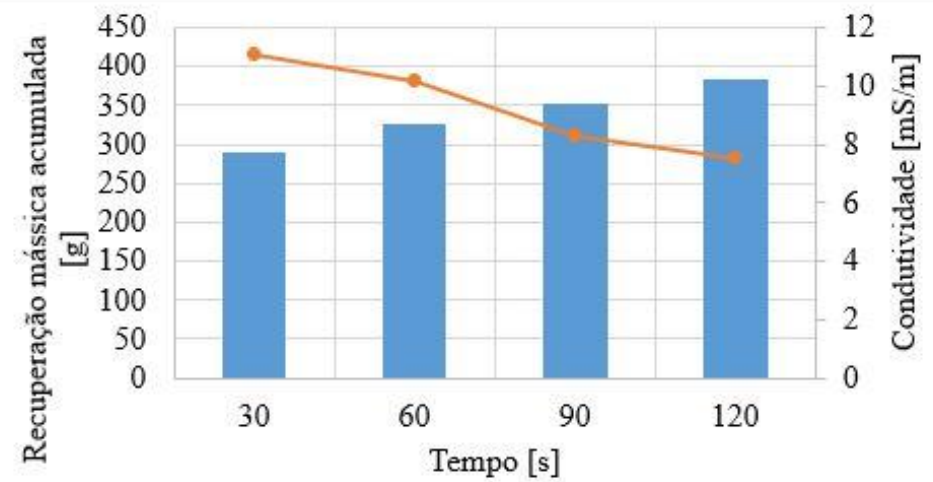

Recuperação mássica [sólidos + água] $\multimap$ Condutividade

Fonte: autoria própria.

Já na "Figura 6" estão os valores de fração de ar calculados no tempo de operação. Embora haja leves alterações entre os dois resultados, percebe-se a tendência deles de convergirem aos 
mesmos valores. Percebe-se que a fração volumétrica de ar aumenta à medida que há expulsão da polpa da célula.

Figura 6 - Evolução da fração volumétrica de ar com o decorrer da operação.

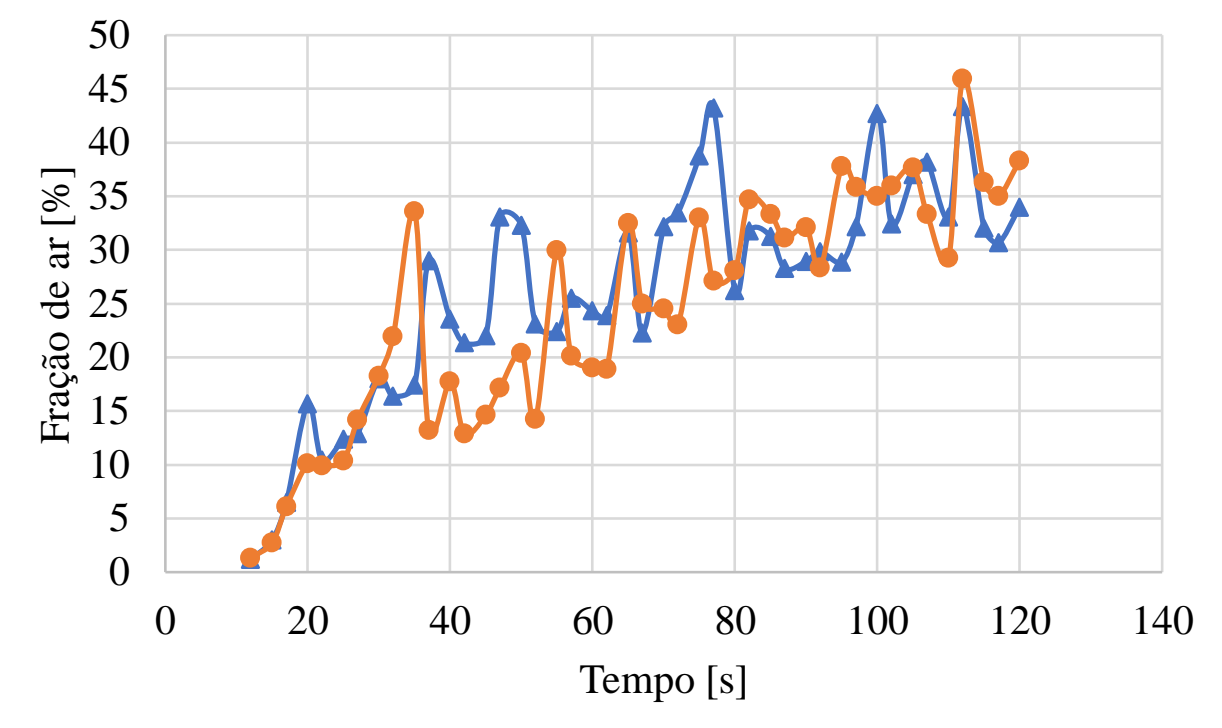

Fonte: autoria própria.

\section{CONSIDERAÇÕES FINAIS}

A fração volumétrica de ar, ou holdup, é uma variável muito importante na flotação mineral. Utilizando-se da equação de Maxwell, é possível calcular seu valor com base em leituras de condutividade da polpa com e sem aeração.

Além de se obter a fração volumétrica de gás, é possível utilizar a leitura de condutividade para medir a velocidade de bolhas em ascensão numa coluna de flotação (SHEN; FINCH, 1996), água de lavagem na flotação (MALDONADO et al., 2008) ou mesmo da fração de sólidos ou de líquidos num sistema ternário (MALDONADO et al., 2017).

Com isso em mente, propôs-se aqui a elaboração de um sistema de leitura de condutividade de polpas minerais com uso da placa Arduino aliado à construção de sonda condutivimétrica de baixo custo.

O sistema apresentou bom desempenho, sendo interessante tanto no sentido de implementar a automação de operações laboratoriais com opções de baixo custo, como por garantir aos estudantes aliar conhecimentos simples de eletrônica e programação à conceitos específicos operacionais.

\section{Agradecimentos}

Os autores agradecem ao CNPq, CAPES, FAPEMIG e UFOP, pelo apoio financeiro para elaboração do trabalho.

\section{REFERÊNCIAS}

AQUINO, J. A. DE; OLIVEIRA, M. M. L. DE; FERNANDES, M. D. Flotação em coluna. In: Tratamento de Minérios. $5^{\mathrm{a}}$ ed. Rio de Janeiro: CETEM/MCT, 2010. p. 515-556.

BAHRI, Z.; SHAFAEI, S. Z.; KARAMOOZIAN, M. Investigation of effective parameters on 
the gas holdup in column flotation of a coal tailing sample. International Journal of Coal Preparation and Utilization, v. 33, n. 2, p. 47-58, 2013.

BANZI, M.; SHILOH, M. Primeiros Passos com Arduino. 2. ed. [s.1.] Novatec, 2015.

BOYER, C.; DUQUENNE, A. M.; WILD, G. Measuring techniques in gas-liquid and gasliquid-solid reactors. Chemical Engineering Science, v. 57, n. 16, p. 3185-3215, 2002.

DFROBOT. DFRobot Gravity: analog electrical conductivity sensor. Disponível em: <https://www.dfrobot.com/product-1123.html>. Acesso em: 29 jul. 2020.

JENA, H. M.; ROY, G. K.; MEIKAP, B. C. Prediction of gas holdup in a three-phase fluidized bed from bed pressure drop measurement. Chemical Engineering Research and Design, v. 86, n. 11, p. 1301-1308, 2008.

MALDONADO, M. et al. On-line bias estimation using conductivity measurements. Minerals Engineering, v. 21, n. 12-14, p. 851-855, 2008.

MALDONADO, M.; Pinto, A.; Gomez, C.O. e Becerra-Yoma, N. Electrode arrangements for continuous measurement of dispersion conductivity in lab dlotation cells. Minerals Engineering, v. 111, p. 1-4, 2017.

OLIVEIRA, J. A.; LUZ, J. A. M.; FERREIRA, E. E. Grau de saponificação de óleos vegetais na flotação seletiva de apatita de minério carbonatítico. Rem: Revista Escola de Minas, v. 59, n. 4, p. 385-390, 2006.

PAN, H.; Li, Y.; Li, N. et al. Study on gas holdup in a fluidized flotation column from bed pressure drop. Energy Sources, Part A: Recovery, Utilization and Environmental Effects, v. 40, n. 14, p. 1693-1700, 2018.

SASTRI, S. R. S. Column flotation: theory and practice. Workshop on Froth Flotation: Recent Trends. Anais...Jamshedpur: National Metallurgical Laboratory, 1998

SHEN, G.; FINCH, J. A. Bubble swarm velocity in a column. Chemical Engineering Science, v. 51, n. 14, p. 3665-3674, 1996.

TAVERA, F. J.; ESCUDERO, R.; FINCH, J. A. Gas holdup in flotation columns: Laboratory measurements. International Journal of Mineral Processing, v. 61, n. 1, p. 23-40, 2001.

TAVERA, F. J.; GOMEZ, C. O.; FINCH, J. A. Estimation of gas holdup in froths by electrical conductivity: Application of the standard addition method. Minerals engineering, v. 11, n. 10, p. 941-947, 1998.

TEIXEIRA, A. V. S.; SANTOS, A. V. Construção de medidor de condutividade digital portátil e de baixo custo. VIII CONNEPI - Congresso Norte Nordeste de Pesquisa e Inovação. Anais...Salvador, Bahia: 2013

TORRES, G. Fundamentos de Eletrônica. Rio de Janeiro: Axcel Books do Brasil Editora, 2002.

URIBE-SALAS, A.; GOMEZ, C. O.; FINCH, J. A. A conductivity technique for gas and solids holdup determination in three-phase reactors. Chemical Engineering Science, v. 49, n. 1, p. 1-10, 1994.

XU, M.; FINCH, J. A.; HULS, B. J. Measurement of radial gas holdup profiles in a flotation column. International Journal of Mineral Processing, v. 36, n. 3-4, p. 229-244, 1992.

ZHU, J. Y.; TAN, F.; GLEISNER, R. Monitoring liquid and solid content in froth using conductivity. Progress in Paper Recycling, v. 14, n. 4, p. 21-29, 2005. 


\title{
FLOTATION FROTHS CHARACTERIZATION THROUGH ARDUINO
}

\begin{abstract}
Flotation is an operation widely used in mineral processing. It selectively separates minerals through superficial modifications by surfactants action. It is carried out in a pulp with aeration, so that the artificially hydrophobized particles adheres to the air bubbles. They are important variables and its control can be done with determination of the hold up, being one of the most important variables. Its determination is usually made by measuring the pressure difference between two points of the equipment, although it can also be done through conductivity measurements. Thus, a low cost system was developed to measure electrical conductivity in a flotation system, using the Arduino platform. The results showed that the system proved to be effective for conductivity measurements, being a proposal both to implement multidisciplinary learning and to acquire data in the laboratory at low cost.
\end{abstract}

Keywords: Arduino. flotation. holdup, electrical conductivity, low cost. 